\title{
Journal level metrics
}

Public Domain

\section{Source}

Open Research Glossary

Metrics that apply to all papers published within a journal. A common example is Thomson Reuters' journal impact factor. 